\begin{tabular}{|l|l|l|l|}
\hline Eiszeitalter u. Gegenwart & $\mathbf{2 9}$ & $\begin{array}{l}71-76 \\
4 \mathrm{Abb} .\end{array}$ & Hannover 1979 \\
\hline
\end{tabular}

\title{
Zur Entstehung des Bodenseebeckens
}

\author{
Albert Schreiner \\ Lake, Glacial erosion, River erosion, Palaeorelief (River deviation), Subsidence, \\ Sedimentation rate, Miocene-Pleistocene, Lake of Constance
}

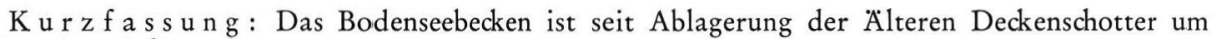
$700 \mathrm{~m}$ eingetieft worden.

Die Entstehung des Beckens wird dem Zusammenwirken von fluviatiler und glazialer Erosion zugeschrieben, wie es schon von PENCK (1909: 420) skizziert wurde. Der starke Anteil der fluviatilen Erosion $(350 \mathrm{~m})$ wird auf die Umlenkung des Alpenrheins zum Hochrhein-Oberrhein zurückgeführt. Geringe tektonische Absenkungen im Verlauf älterer Bruchlinien werden zur Erklärung der NW-SE-Richtung der westlichen Seearme herangezogen.

\section{[On the Excavation of the Lake of Constance Basin]}

A bstract: The trough of the Lake of Constance has been hollowed out until to a depth of about $700 \mathrm{~m}$ after the "Older Deckenschotter" had been deposited.

As PENCK (1909: 420) has already indicated, the excavation of the hollow is considered to have been caused by both the erosion of glaciers as well as of streams.

The major portion of fluvial erosion is supposed to be due to the deviation of the Alpenrhein, which thus got a westerly way in the direction to Hochrhein-Oberrhein.

Slight tectonic subsidences are thought to have been causing the NW-SE-course of the western branches of the lake.

Der Bodensee, Deutschlands größter See, hat heute eine Länge von rund $60 \mathrm{~km}$, eine Breite von $10 \mathrm{~km}$, und eine größte Wassertiefe von $250 \mathrm{~m}$ (Wasserspiegel heute bei $395 \mathrm{~m}$ üb. NN). Seine größte Ausdehnung hatte der Bodensee im Spätglazial nach dem Abschmelzen des würmeiszeitlichen Rheingletschers. Er reichte, vielleicht durch Schwellen unterbrochen, weit in das Tal des Alpenrheins hinein. Die Vorstellung eines durchgehenden „Rheinsees“ bis oberhalb von Chur mit Verbindungen zum Walensee-Zürichsee (Albert HeIm 1919: 400, Georg WAGNER 1962: 4) ist nach nicht veröffentlichten Bohrergebnissen nicht mehr aufrecht zu erhalten (Diskussionsbeitrag HANTKE).

\section{Längsschnitt durch das Bodenseebecken (Abb. 1)}

Der Längsschnitt zeigt das Relief der Quartärbasis und die Mächtigkeit der Quartärsedimente. In der Erdölaufschlußbohrung Dornbirn 1 liegt die Quartärbasis nach Huf (1963) in $337 \mathrm{~m}$ Tiefe (= $77 \mathrm{~m}$ üb. NN). Der größte Teil des Quartärs, bis in $300 \mathrm{~m}$ Tiefe, besteht aus holozänen Seetonen; Moräne fehlt. Der Bohrpunkt liegt etwas randlich und es ist möglich, daß das Quartär in der tiefsten Talrinne noch tiefer reicht.

Für die Angaben zur Eintiefung in der Seemitte wurden die Ergebnisse reflexionsseismischer Messungen des Niedersächsischen Landesamtes für Bodenforschung (MülleR \& GeEs 1968) herangezogen. Danach sollen unter dem Seeboden des Obersees noch $150 \mathrm{~m}$ Seesedimente und Moränen liegen, was eine Quartärbasis von $0 \mathrm{~m} \pm \mathrm{NN}$ ergibt.

*) Anschrift des Verfassers: Dr. A. S ch r e in e r, Geologisches Landesamt Baden-Württemberg, Albertstraße 5, D-7800 Freiburg i. Br. 
Die Thermalwasserbohrung Konstanz mit 200 m pleistozänen Kiesen und Moränen (BüchI et al. 1976) zeigt das Ansteigen der Quartärbasis vom Oberseebecken nach W an. Die von BücHI et al (1976: 26) gegebene Gliederung der erbohrten Quartärschichten von Rißmoräne bis Spätwürmmoräne ist möglich, aber nicht $z$ wingend, solange gesicherte $\mathrm{Da}-$ tierungen fehlen. Deshalb können auch die Fragen, ob im Bodenseebecken rißeiszeitliche Sedimente liegen, oder ob der würmeiszeitliche Gletscher die größte Austiefung des Bekkens geschaffen hat, noch nicht beantwortet werden.

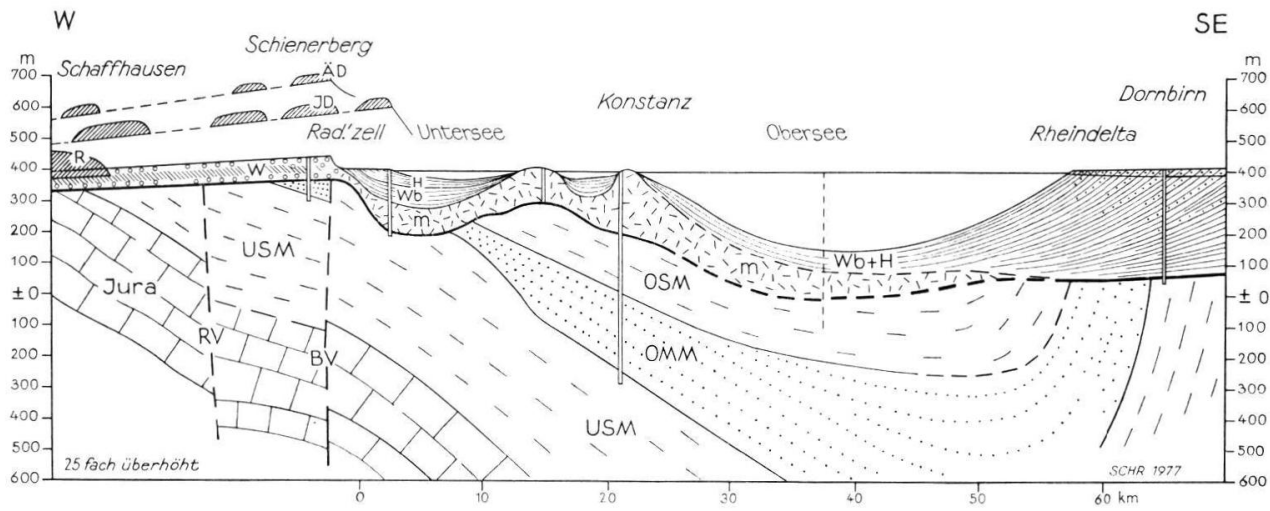

Abb. 1: Längsschnitt längs der Mittellinie durch das Bodenseebecken von Schaffhausen bis in das Rheindelta bei Dornbirn. $\mathrm{H}=$ holozäne Sedimente (Seetone, Sande, Kiese); Wb = würmeiszeitliche Seesedimente (Beckentone u. -sande); $W=$ würmeiszeitliche Kiese und Moränen, an der Basis „Rinnenschotter"; $\mathrm{m}=$ Moränen und Kiese, würmeiszeitlich und vielleicht älter; $\mathrm{R}=$ rißeiszeitliche Kiese und Moränen; JD = Jüngere Deckenschotter (Mindel?); $\mathrm{AD}=$ Ältere Deckenschotter (Günz?); OSM = Obere Süßwassermolasse; OMM = Obere Meeresmolasse; USM = Untere Süßwassermolasse; RV = Randen-Verwerfung; BV = Buchberg-Verwerfung. Weitere Erklärungen im Text.

Wasserbohrungen auf der Insel Reichenau (nicht veröffentlicht) mit $91 \mathrm{~m}$ Pleistozän und eine Forschungsbohrung bei Radolfzell (MüLleR, G. et al 1967) mit $200 \mathrm{~m}$ Seesedimenten, Moränen und Kieslagen beleuchten die Verhältnisse am Untersee. Die Geröllzusammensetzung auch der tiefsten Kieslage in $196 \mathrm{~m}$ Tiefe bietet keinen sicheren Hinweis, daß hier ältere als würmeiszeitliche Quartärsedimente erbohrt wurden (STAEsche 1972: 47).

Es ist bemerkenswert, daß die fast bis in $200 \mathrm{~m}$ Tiefe liegenden Kiese in den Bohrungen bei Konstanz und Radolfzell keine normale Vorflut hatten, denn die tiefste Rinnenbasis im W liegt $150 \mathrm{~m}$ höher. Es dürfte sich um subglazial abgelagerte Kiese handeln (SCHREINER 1968: 92).

Von Radolfzell zum Hochrhein bei Schaffhausen wurde in Abbildung 1 der Weg eingezeichnet, den die Rinnenschotter und die Schmelzwässer beim Hochstand der Würmeiszeit genommen haben. Der heutige Rheinlauf von Stein a. Rh. nach Schaffhausen wurde erst ab dem Stadium 7 (Terrasse Singen-Ramsen) von dem Schmelzwasserstrom des Rheingletschers durchflossen.

Aus der Höhenlage der Deckenschotter, die vom westlichen Bodenseegebiet nach W ziehen, ist die Eintiefung des Bodenseebeckens seit Ablagerung der Deckenschotter abzulesen: Von den Älteren Deckenschottern auf dem Schienerberg bei $700 \mathrm{~m}$ üb. NN bis zur tiefsten Quartärbasis im Bodenseebecken ergibt sich eine Gesamteintiefung von $700 \mathrm{~m}$; im Unterseebecken sind es $500 \mathrm{~m}$. 


\section{Fluviatile Eintiefung}

An der Treppe der fluvioglazialen Schotter am westlichen Bodensee von den Älteren Deckenschotter bis zu den Rinnenschottern, die als würmeiszeitliche Vorstoßschotter aufgefaßt werden (SCHREINER 1968: 83), ist eine starke, vorwiegend fluviatile Erosion abzulesen. Sie beträgt rund $350 \mathrm{~m}$ und damit die Hälfte der Gesamteintiefung des Bodenseebeckens. Die Rinnen der Jüngeren Deckenschotter liegen schon $100 \mathrm{~m}$ tiefer und die Basis der Rinnenschotter bei Singen liegt $350 \mathrm{~m}$ tiefer als die Alteren Deckenschotter auf dem Schienerberg. Die entscheidende Ursache für die starke, fluviatile Tiefenerosion ist die bekannte Umlenkung des Alpenrheins nach W zum Hochrhein-Oberrhein (Abb. 2 mit Bildtext). Die neue Erosionsbasis, die Oberrheinebene, lag 200 bis $300 \mathrm{~m}$ tiefer als die frühere Erosionsbasis, die Donau. Die Folge war die starke Tiefenerosion auf der Strecke Alpenrhein-Bodensee-Hochrhein. Die Erosionswirkung wurde ermöglicht durch die Hebungstendenz des Alpenvorlandes und verstärkt durch das kaltzeitliche Klima.

Die Umlenkung des Alpenrheins nach W ist auch die Ursache für die W-Richtung des Bodensees, im Gegensatz zur N-Richtung vieler Seen im Voralpenland.

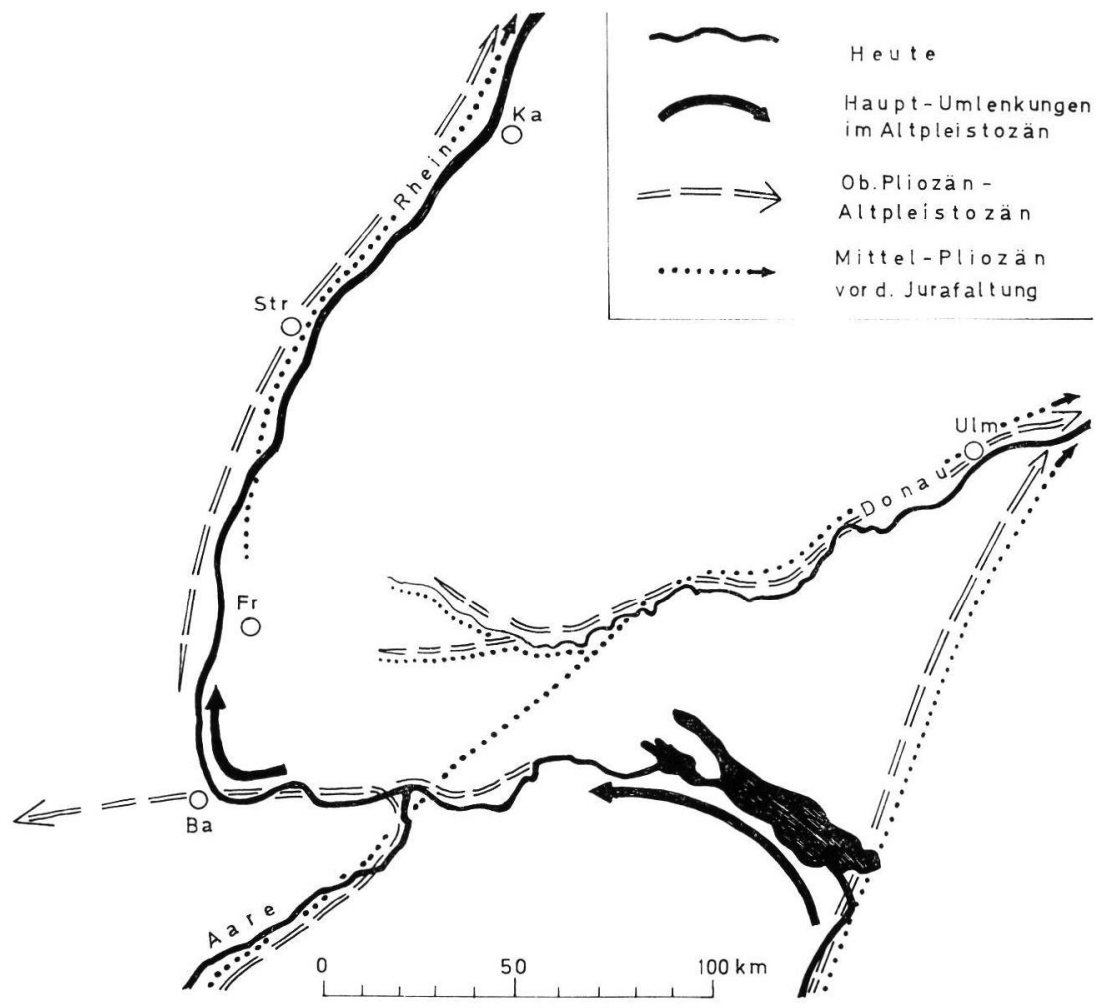

Abb. 2: Umlenkung des Alpenrheins nach W (nach Liniger 1962, stark vereinfacht). Das pliozäne Flußnetz der Aare-Donau erfuhr wesentliche Wandlungen. Im Zug der oberpliozänen Jurafaltung und Schwarzwaldhebung wurde die Aare nach W umgelenkt (Sundgauschotter). Zu Beginn des Pleistozäns lief dann die Aare nach $\mathrm{N}$ in den sich senkenden Oberrheingraben über. Wahrscheinlich infolge Anzapfung durch einen östlichen Nebenfluß der Aare und durch fluvioglaziale Aufschotterungen im westlichen Bodenseegebiet bei den ersten Vereisungen brach der ursprünglich nach $\mathrm{N}$ zur Donau fließenden Alpenrhein nach $\mathrm{W}$ durch, womit der heutige Lauf AlpenrheinBodensee-Hochrhein-Oberrhein geschaffen wurde. 


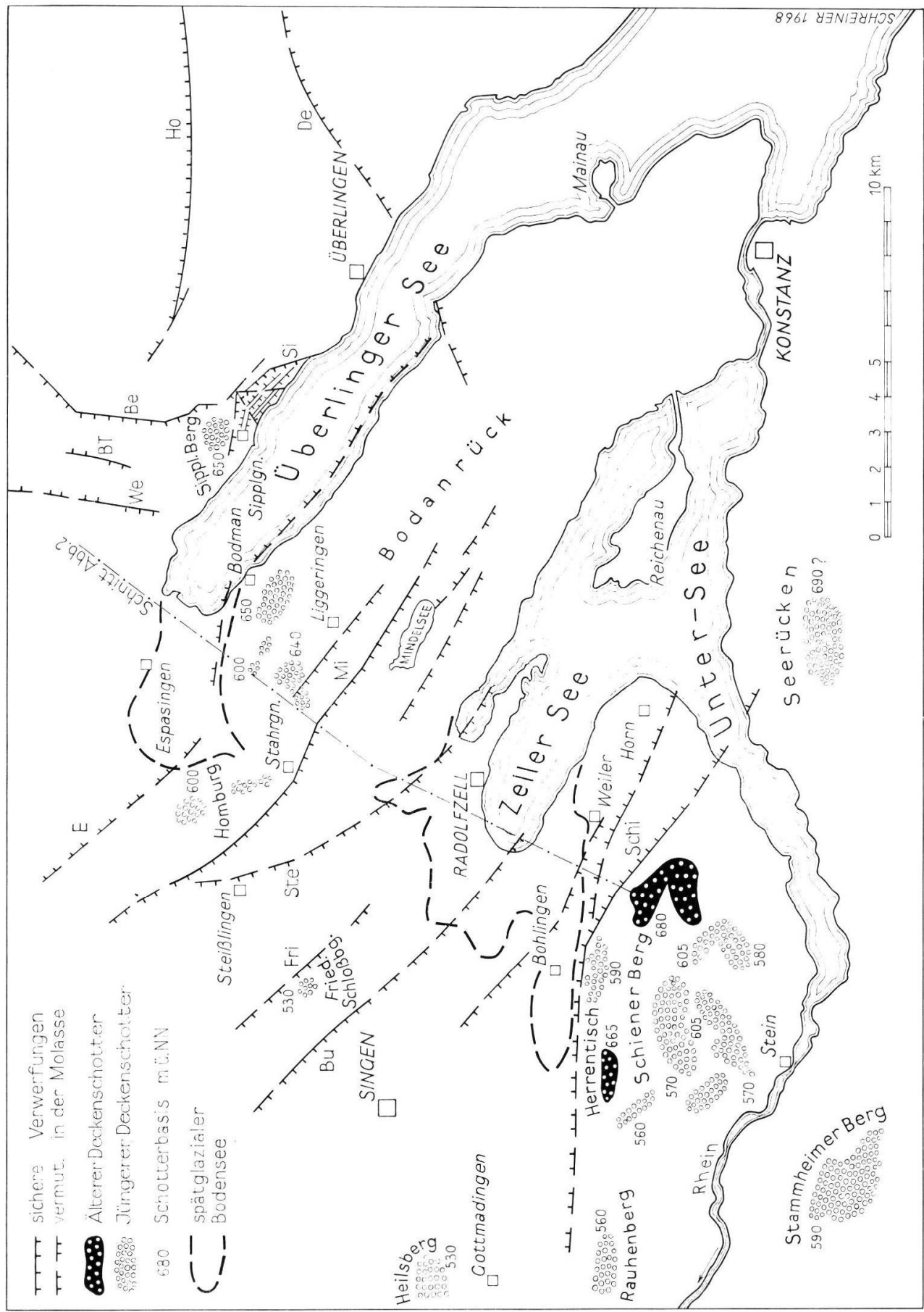

Abb. 3: Westlicher Bodensee mit Verwerfungen in den Schichten des Tertiärs; und Deckenschottervorkommen. Der Schotterzug vom Sipplinger Berg über Bodanrück-Homberg zum Heilsberg überquert die NW-SE-streichenden Verwerfungen. $\mathrm{Bu}$ bis $\mathrm{De}=$ Bezeichnung der Verwerfungen (SCHREINER 1970: 152). Neu: E. Eigeltinger Verwerfung. 


\section{Glaziale Erosionen}

Die tiefen fluviatilen Rinnen wurden von dem in den Kaltzeiten weit ins Vorland vorstoßenden Rheingletschern ausgeweitet und übertieft. Es ist bislang nicht bekannt, wie tief das Bodenseebecken von dem Gletscher z. B. der Mindeleiszeit oder der Rißeiszeit ausgefurcht wurde (siehe auch Abschnitt 1). Wahrscheinlich ist, daß der Wasserspiegel des Bodensees nach der Rißeiszeit um ungefähr $50 \mathrm{~m}$ tiefer lag als heute, denn die Basis der würmeiszeitlichen Vorstoßschotter bei Radolfzell - Singen liegt bei $350 \mathrm{~m}$ üb. NN (SCHreINer 1968: Abb. 3).

\section{Tektonik}

Die Rolle der Tektonik für die Entstehung des Bodenseebeckens ist besonders wegen des grabenartig aussehenden Überlinger Sees in den Vordergrund gestellt worden (DEECKE 1916: 676). Tektonische Senkungen im Quartär, die zur Entstehung des Seebeckens geführt oder beigetragen haben, sind sehr schwer nachzuweisen. Bohrungen im Verlandungsgebiet am NW-Ende des Überlinger Sees auf die Grenze Tertiär/Jura haben gerade in diesem, für einen Graben besonders verdächtigen Gebiet, zumindest am Nordrand des Beckens das Nichtvorhandensein einer Grabenrandverwerfung erwiesen (SCHREINER 1975: 61).

Am Südrand des Überlinger Sees bei Bodman kann aus der Lagerung der Molasseschichten eine Verwerfung von 40 bis $50 \mathrm{~m}$ Sprunghöhe ermittelt werden, und am Untersee ziehen NW-SE-verlaufende Bruchlinien der Lenzkirch-Bonndorfer Grabenzone in den Bodensee hinein (Schreiner 1970: Beil. 2).

Das Wesentliche für die Entstehung des Bodenseebeckens ist aber, daß die zahlreichen Verwerfungen, die bei der geologischen Aufnahme des westlichen Bodenseegebietes festgestellt wurden (Schreiner 1970), die Schichten des Jura und des Tertiärs versetzen. Die altpleistozänen Deckenschotter hingegen ziehen, abgesehen von einer Ausnahme, ohne Störung ihre Gefällslinie über die im älteren Unterbau festgestellten Verwerfungen hinweg. Die Verwerfungen sind demnach älter, wahrscheinlich obermiozänen bis pliozänen Alters. Die erwähnte Ausnahme ist der Jüngere Deckenschotter auf dem Friedinger Schloßberg bei Singen, der um 20 bis $30 \mathrm{~m}$ zu tief liegt (Abb. 4). Hier hat im Bereich eines

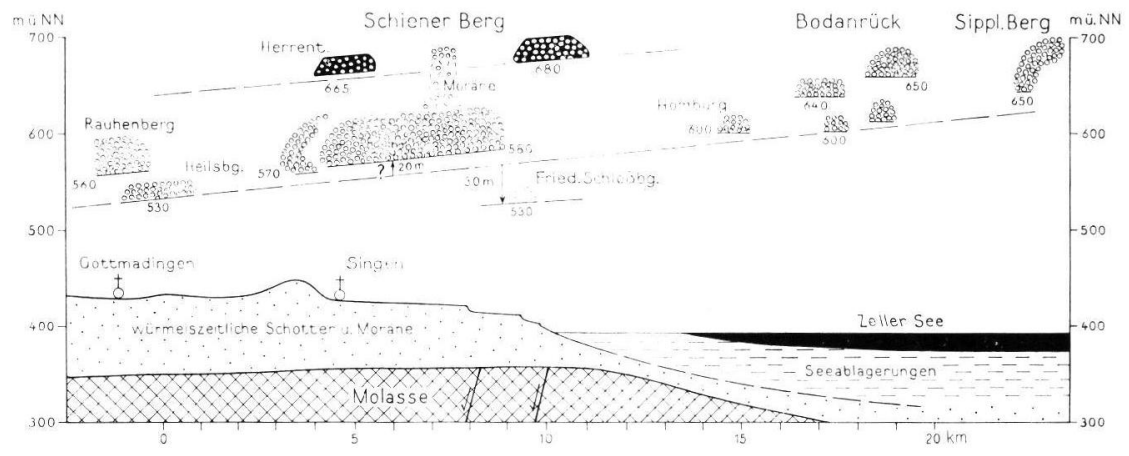

\$28: AltererDeckenschotter

$\frac{8000}{80.00} 50$ Jüngerer Dechenschotter

Abb. 4: Deckenschotter im westlichen Bodenseegebiet. Auf einen Schnitt von Sipplingen bis Gottmadingen projeziert. Der Jüngere Deckenschotter des Friedinger Schloßberges ist gegenüber der Schotterbasis der tieferen Schottervorkommen um 30 m abgesenkt. 
im Tertiär angelegten Grabens nach Ablagerung der Deckenschotter eine Nachsenkung stattgefunden. Der Betrag von $30 \mathrm{~m}$, auch wenn er weiter im SE auf $100 \mathrm{~m}$ ansteigen sollte, reicht bei weitem nicht aus, um die Gesamteintiefung des Bodenseebeckens von $700 \mathrm{~m}$ zu erklären.

Es ist jedoch offensichtlich, daß die NW-Richtung des Uberlinger und des Zeller Sees tektonisch bedingt sind, in der Weise, daß die fluviatile Erosion nach dem Altpleistozän den neu entstandenen tektonischen Tieflagen folgte und damit die Richtung für die nachfolgende Gletschererosion gewiesen hat.

\section{Impakt im Bodenseegebiet?}

Funde von Blöcken aus Malmkalk mit Shatter-Cone und von Splittern exotischer Gerölle in der Oberen Süßwassermolasse der Ostschweiz haben zu der Annahme geführt, daß zur gleichen Zeit wie im Nördlinger Ries auch im Bereich des heutigen Obersees ein großer Meteorit eingeschlagen habe (Hofmann 1973). Es ist jedoch darauf hinzuweisen, daß aufgrund von Erdölbohrungen nördlich des Obersees am Ort des vermuteten Impakts eher Jura in helvetischer Fazies und nicht in schwäbischer Fazies (Weißjura) wie in den Blöcken zu erwarten ist.

Mit der Entstehung des Bodensees dürfte der Meteoritenkrater, falls er sich als richtig herausstellen sollte, aber nicht in Verbindung gebracht werden, denn der Krater wäre von den alpinen Schwemmfächern rasch verfüllt und mit einigen $100 \mathrm{~m}$ Sedimenten der höheren Oberen Süßwassermolasse (Mergel, Sandsteine und Konglomerate ähnlich wie am Pfänder) überdeckt worden. Erst danach hätte dann die plio-pleistozäne Abtragung und Ausräumung des Bodenseebeckens begonnen.

\section{Schriftenverzeichnis}

BüChI, U. P., Schlanke, E. \& Müller, E. (1976): Zur Geologie der Thermalwasserbohrung Konstanz und ihre sedimentpetrographische Korrelation mit der Erdölbohrung Kreuzlingen. Bull. Ver. schweiz. Petrol.-Geol. u. Ing., 42, 103: 25-33; Basel.

DeEcke, W. (1916): Geologie von Baden, 1. Teil, 782 S., Berlin (Bornträger).

Heim, Albert (1919): Geologie der Schweiz, Bd. I, 704 S., Leipzig (Tauschnitz).

Hofmann, F. (1973): Horizonte fremdartiger Auswürflinge in der ostschweizerischen Oberen Süßwassermolasse und Versuch einer Deutung ihrer Entstehung als Impaktphänomen. Eclogae geol. Helv., 66/1: 83-100; Basel.

HuF, W. (1963): Die Schichtenfolge der Aufschlußbohrung „Dornbirn 1“ (Vorarlberg, Ósterreich). - Bull. Ver. schweiz. Petrol.-Geol. u. -Ing., 29, 77: 9-10; Basel.

Liniger, H. (1966): Das Plio-Altpleistozäne Flußnetz der Nordschweiz. - Regio Basiliensis, 7: 158-177; Basel.

MüLleR, G. \& GeEs, R. A. (1968): Erste Ergebnisse reflexionsseismischer Untersuchungen des Bodensee-Untergrundes. - N. Jb. Geol. Paläont., Mh., 6: 364-369; Stuttgart.

- , Schreiner, A. \& Staesche, W. ( ): Kurzprofile der wissenschaftlichen Bohrungen „Bodensee DFG 1 und 2“. - Naturwiss., 54: 87-88; Berlin, Heidelberg.

Penck, A. (1909): in Penck \& Brückner: Die Alpen im Eiszeitalter, 2. Band, 716 S., Leipzig (Tauschnitz).

Schreiner, A. (1968): Eiszeitliche Rinnen und Becken und deren Füllung im Hegau und westlichen Bodenseegebiet. - Jh. Geol. Landesamt Baden-Württemberg, 10: 79-104; Freiburg i. Br.

- (1970): Erläuterungen zur geologischen Karte des Landkreis Konstanz mit Umgebung 1:50000. - Geol. Landesamt Baden-Württemberg: 286 S.; Freiburg i. Br.

- (1975): Zur Frage der tektonischen oder glazigen-fluviatilen Entstehung des Bodensees. Jber. u. Mitt. oberrh. geol. Ver., N. F. 57: 61-75; Stuttgart.

Staesche, W. (1972): Mineralogisch-sediment-petrographische und isotopengeochemische Untersuchungen an den Kernproben der wissenschaftlichen Bohrungen „Bodensee DFG I und II" im Verlandungsgebiet des Untersees (Bodensee). - Diss. Univ. Heidelberg: 72 S.; Heidelberg. - [Unveröff.]

WaGner, Georg (1972): Zur Geschichte des Bodensees. - Jb. 1962, 27, Ver. z. Schutz der Alpenpflanzen u. -Tiere: $17 \mathrm{~S}$.; München. 